\title{
Robust Multipath Exploitation Radar Imaging in Urban Sensing Based on Bayesian Compressive Sensing
}

\author{
Qisong Wu, Yimin D. Zhang, Moeness G. Amin, and Fauzia Ahmad \\ Center for Advanced Communications, Villanova University, PA 19085, USA
}

\begin{abstract}
In through-the-wall radar imaging applications, exploitation of group sparsity of the targets under multipath propagation allows high-resolution ghost-free imaging. However, such multipath exploitation schemes may suffer from imperfect knowledge of the surrounding scatterers, such as interior walls. In this paper, a novel two-stage Bayesian compressive sensing approach is proposed for joint scene reconstruction and wall location estimation. The proposed method is capable of not only acquiring enhanced images by exploiting multipath propagation, but also estimating wall locations with high accuracy. In addition, compared to computationally demanding genetic approaches, the proposed method achieves robust imaging with a low complexity.
\end{abstract}

Index Terms-Through-the-wall radar imaging, multipath exploitation, Bayesian compressive sensing, group sparsity.

\section{INTRODUCTION}

Through-the-wall radar imaging (TWRI) is an emerging technology that attracts increasing interests due to its capability to reveal stationary or moving targets of interest behind an opaque obstacle [1-5]. A major challenge in TWRI is the multipath originating from multiple reflections of electromagnetic waves off the targets in conjunction with the walls. Such multipath reflections give rise to false targets or "ghosts" at positions other than those of the actual targets. These ghost targets clutter the imaged scene and compromise target detection [6]. If precise prior knowledge of the room layout is available, the additional energy contained in the multipath returns can be used to improve the imaging performance and target detection probability [7-9]. However, it is usually difficult to obtain accurate locations of the interior walls a priori in practical operational scenarios. Therefore, it is imperative to develop robust multipath exploitation techniques that can provide accurate imaging in the presence of wall location uncertainties.

Earlier works were proposed to effectively mitigate the effects of multipath propagation [3, 4]. More recent works are based on multipath exploitation using back-projection (BP) $[6,7]$ and compressive sensing (CS) $[8,10]$ techniques for target signal enhancement. The latter schemes take advantage of the group sparsity of the targets across the various multipath returns. However, the underlying assumption of perfect knowledge of the secondary scatterers remains the basis for all of the existing approaches. An alternate approach based on CS reconstruction without the prior knowledge of the building layout has been proposed in [11] to suppress the multipath return energy. A multipath exploitation scheme that reconstructs the through-the-wall image and estimates the wall locations under wall position uncertainties using a nested optimization approach has been proposed in [12], but is computationally demanding due to the non-convexity of the minimization step for wall location estimation.

In this paper, a two-stage imaging scheme, which applies a complex multi-task Bayesian compressive sensing (CMTBCS) [13] for target imaging and maximum likelihood estimation for wall positions, is proposed. Bayesian compressive sensing techniques are known to provide robustness for highresolution signal reconstruction compared to conventional CS approaches due to their insensitivity to dictionary coherence [13-17]. We assume interior walls to be either parallel or perpendicular to the front exterior wall, with only limited information on wall positions. We exploit the fact that the reconstructed targets reflected from the direct path would not suffer from wall position uncertainties. As such, a common target support can be extracted from images obtained from the CMT-BCS algorithm with a few hypothetical wall positions. The accurate wall positions can then be estimated by a maximum likelihood estimator. Finally, the additional energy contained in the multipath returns can be used for effective target signal enhancement. The proposed two-stage method enables ghost-free image reconstruction of the target scene under wall position uncertainties with a low computational complexity. We evaluate the performance of the proposed method using numerical simulations.

Notations: We use lower-case (upper-case) bold characters to denote vectors (matrices). In particular, $\mathbf{I}_{N}$ denotes the $N \times N$ identity matrix. $p(\cdot)$ denotes the probability density function (pdf), and $\mathcal{N}(x \mid a, b)$ denotes that random variable $x$ follows a Gaussian distribution with mean $a$ and variance $b$, $\lceil x / y\rceil$ denotes the modulus after $x$ over $y$, and $\lfloor x / y\rfloor$ denotes the remainder after $x$ over $y$. In addition, $(\cdot)^{T}$ denotes transpose. $\operatorname{Re}(x)$ and $\operatorname{Im}(x)$, respectively, represent the real and imaginary parts of the complex $x$. $\circ$ denotes the element-wise (Hadamard) multiplication, and $\otimes$ is a Kronecker product.

\section{Signal Model}

The signal model is formulated under the assumptions of monostatic stepped-frequency operation and a scene of stationary targets. $N$ wideband transceivers are considered, which are arranged as a uniform linear array (ULA) with element positions $x_{n}=(n-1) d, n=1, \cdots, N$, where $d$ is the inter-element spacing. Let the $n$th transceiver transmit a stepped-frequency signal consisting of $M$ frequencies, $f_{m}$, $m=1, \cdots, M$, which are uniformly spaced over the signal bandwidth $B$. We consider a two-dimensional (2-D) image of the target scene in the downrange and crossrange directions, which is discretized into $N_{x} \times N_{y}$ pixels, with $N_{x}$ and $N_{y}$ 
respectively denoting the number of pixels in crossrange and downrange within the region of interest. Under monostatic operation, the target returns can be expressed in terms of the frequency index $m$ and antenna position index $n$ as [8],

$$
y(m, n)=\sum_{p=0}^{N_{x} N_{y}-1} w_{p} \exp \left(-j 2 \pi f_{m} \tau_{p n}\right),
$$

where $w_{p}$ is the complex reflectivity of the $p$ th pixel, and $\tau_{p n}$ represents the round-trip propagation delay between the $p$ th pixel and the $n$th transceiver.

The target scene usually has sparse occupancy, i.e., most of the $N_{x} \times N_{y}$ pixels have negligible scattering coefficients. Let $P$ be the scene sparsity, or the number of nonzero pixels in the entire image with $N_{x} \times N_{y}$ pixels. All $M N$ measurements $y(m, n)$ for $m=1, \cdots, M$ and $n=1, \cdots, N$ are stacked into a single column vector to obtain the measurement data vector $\mathbf{y}=[y(1,1), \cdots, y(1, N), \cdots y(M, N)]^{T} \in \mathcal{C}^{M N}$. As such, eq. (1) takes the form

$$
\mathbf{y}=\mathbf{\Phi} \mathbf{w}
$$

where the $(i, l)$ th element of the sensing matrix $\Phi \in$ $\mathcal{C}^{M N \times N_{x} N_{y}}$ is given by

$$
[\boldsymbol{\Phi}]_{i l}=\exp \left(-j 2 \pi f_{m} \tau_{l n}\right)
$$

for $i \in\{1, \cdots, M N\}, m=\lceil i / M\rceil$, and $n=\lfloor i / M\rfloor$, and $\mathbf{w}=$ $\left[w_{1}, \cdots, w_{N_{x} \times N_{y}}\right]^{T} \in \mathcal{C}^{N_{x} N_{y}}$ represents the scene reflectivity vector. The vectorized measurement model in eq. (2) will serve as the linear measurement equation for the CS-based target image reconstruction.

\section{A. Interior Wall Multipath}

In TWRI, multipath propagation corresponds to signal paths to and from the target which involve reflections at one or more secondary reflectors, such as walls. The dominant multipath component corresponds to a bistatic scattering scenario, wherein the transmitted signal propagates directly to the target and then the scattered wave travels back to the transceiver after reflection at an interior wall or vice versa $[6,8]$. If the room geometry is known a priori, the propagation delays associated with the various multipath returns can be readily calculated by ray-tracing $[8,10]$.

The measurements corresponding to the different propagation paths are, in general, not resolvable. Therefore, assuming one direct path and a maximum number of $K-1$ multipath returns, the forward model under multipath propagations renders the received signal vector to be expressed as,

$$
\tilde{\mathbf{y}}=\boldsymbol{\Phi}_{0} \mathbf{w}_{0}+\boldsymbol{\Phi}_{1}\left(x_{w_{1}}\right) \mathbf{w}_{1}+\cdots+\boldsymbol{\Phi}_{K-1}\left(x_{w_{K-1}}\right) \mathbf{w}_{K-1},
$$

where $\boldsymbol{\Phi}_{0} \in \mathcal{C}^{M N \times N_{x} N_{y}}$ denotes the sensing matrix for the direct path as defined in Eq. (3), and $\mathbf{w}_{0} \in \mathcal{C}^{N_{x} N_{y}}$ is the associated complex reflection coefficient vector, whereas $\boldsymbol{\Phi}_{k}\left(x_{w_{k}}\right)$ and $\mathbf{w}_{k}$ with $k \in\{1, \cdots, K-1\}$ are the respective sensing matrix related to the $k$ th multipath with the corresponding wall location of $x_{w_{k}}$ and the associated complex reflection coefficient vector. The elements of $\boldsymbol{\Phi}_{k}\left(x_{w_{k}}\right), k \in$
$\{1, \cdots, K-1\}$ are phase terms, analogous to (3), incorporating the corresponding propagation delays. It should be noted that the complex reflection coefficients $\mathbf{w}_{k}, k=0, \cdots, K-1$, share the same nonzero support across all paths, i.e., they have the same respective positions of nonzero entries, but the exact value of the reflectivity coefficients generally differ for each path.

Rewrite (4) in a compact form as

$$
\begin{aligned}
\tilde{\mathbf{y}} & =\boldsymbol{\Psi}\left(\mathbf{x}_{w}\right) \tilde{\mathbf{w}}+\boldsymbol{\epsilon}, \\
\tilde{\mathbf{w}} & =\left[\mathbf{w}_{0}, \cdots, \mathbf{w}_{K-1}\right]^{T} \in \mathcal{C}^{N_{x} N_{y} K}, \\
\mathbf{w}_{k} & =\left[w_{0, k}, \cdots, w_{N_{x} N_{y}-1, k}\right] \in \mathcal{C}^{1 \times N_{x} N_{y}},
\end{aligned}
$$

where $\boldsymbol{\Psi}\left(\mathbf{x}_{w}\right)=\left[\boldsymbol{\Phi}_{0}, \boldsymbol{\Phi}_{1}\left(x_{w_{1}}\right), \cdots, \boldsymbol{\Phi}_{K-1}\left(x_{w_{K-1}}\right)\right] \in$ $\mathcal{C}^{M N \times N_{x} N_{y} K}$ is the joint sensing matrix, and $\tilde{\mathbf{w}}$ represents the complex reflection coefficient vector. Without loss of generality, a measurement noise vector $\epsilon \in \mathcal{C}^{M N}$ is added in (5).

Within the CS framework, we may perform sparse reconstruction using only a subset of the full measurements. Mathematically, the data reduction operation can be expressed as a downsampling matrix $\mathbf{D} \in \mathcal{R}^{J \times M N}$ acting on the full measurements, where $J \ll M N$ is the number of reduced measurements. For stepped-frequency radar system, a binary measurement matrix $\mathbf{D} \in\{0,1\}^{J \times M N}$ is a reasonable choice $[10,18]$. As such, we acquire an undersampled measurement vector $\mathbf{z} \in \mathcal{C}^{J}$, expressed as

$$
\mathbf{z}=\mathbf{D} \tilde{\mathbf{y}}=\mathbf{D} \boldsymbol{\Psi}\left(\mathbf{x}_{w}\right) \tilde{\mathbf{w}}+\mathbf{D} \boldsymbol{\epsilon}=\mathbf{A}\left(\mathbf{x}_{w}\right) \tilde{\mathbf{w}}+\tilde{\boldsymbol{\epsilon}},
$$

with $\mathbf{A}\left(\mathbf{x}_{w}\right)=\mathbf{D} \Psi\left(\mathbf{x}_{w}\right) \in \mathcal{C}^{J \times N_{x} N_{y}}$ denotes the dictionary matrix, and $\tilde{\boldsymbol{\epsilon}}=\mathbf{D} \boldsymbol{\mathrm { \epsilon }} \in \mathcal{C}^{J}$.

\section{B. Effect of Wall Position Uncertainty}

With the knowledge of precise wall positions, the return signals traveling along each path can be coherently combined by constructing the sensing matrices based on the true wall locations. However, when the information of the wall locations is inaccurate, the difference between the assumed and the actual wall locations will cause significant degradation of the reconstructed image [12]. That is, when the assumed delays do not match the true propagation delays, the reflections under multipath propagation may appear at image pixels different from the true target locations, resulting in ghost targets, as shown in Fig. 1. As the ghost targets from multipath propagations may be reconstructed at different locations, they violate the group sparsity property that relates them to the true target positions. The presence of ghost targets also increase the effective scene sparsity, degrading the efficiency of sparse reconstruction techniques.

In the following section, a novel two-stage scheme which applies the CMT-BCS approach for targets imaging and maximum likelihood estimation for the wall uncertainty, is proposed to jointly estimate true wall positions and acquire high-resolution imaging.

\section{Proposed Algorithm}

We exploit the fact that the target reflections under the direct paths do not depend on the interior wall positions. 


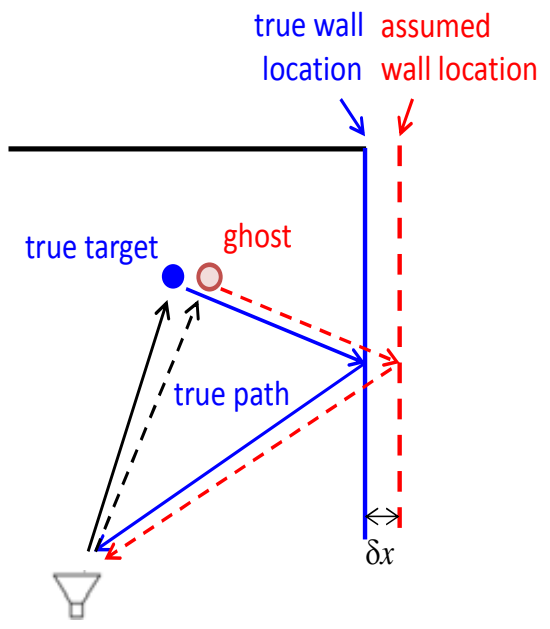

Fig. 1: Multipath propagation related to wall location.

As such, the common support occupied by the true targets can be obtained even under assumed wall locations that are different from the true ones, whereas the ghost targets due to the multipath propagations will appear at diverse positions under wall location inaccuracies.

The proposed robust imaging approach consists of two major stages. In the first stage, multiple images with true as well as ghost targets are obtained by utilizing the CMT-BCS technique under a small number $D$ of different hypothetical wall locations. The pixels of the common nonzero support of these images, which are considered as the true target positions corresponding to the direct path, are then used to estimate the wall locations through maximum likelihood estimation. In the second stage, a high-resolution image is reconstructed through multipath exploitation using the sensing matrix that is constructed with the estimated wall locations. The details of these two stages are provided below.

\section{A. First Stage: Wall Parameter Estimation}

In the first stage, we exploit the CMT-BCS algorithm to recover the scattering vector $\tilde{\mathbf{w}}^{(d)}$, which contains both true targets and ghost targets, for the $d$ th hypothetical wall locations $\mathbf{x}_{w}^{(d)}=\left[x_{w_{1}}^{(d)}, \cdots, x_{w_{K-1}}^{(d)}\right], d=1, \cdots, D$. As mentioned above, the true targets corresponding to the direct paths share the same positions across the $D$ reconstructed images, whereas the ghost targets appear at different positions in each image. Therefore, we extract the scattering vector corresponding to the true targets, $\tilde{\mathbf{w}}$, based on the common support from $D$ sub-images.

We first briefly introduce the CMT-BCS for sparse reconstruction with group sparsity [13], which will be applied separately to each set of hypothetical wall positions. The target reflection coefficient vector at the $p$ th pixel can be defined for all $K$ paths as

$$
\mathbf{w}_{p}=\left[w_{p, 0}, \cdots, w_{p, K-1}\right]^{T} .
$$

We decompose the complex $\mathbf{w}_{p} \in \mathcal{C}^{K}$ into $\check{\mathbf{w}}_{p} \in \mathcal{R}^{2 K}$ as

$$
\check{\mathbf{w}}_{p}=\left[w_{p, 0}^{(R)}, \cdots, w_{p, K-1}^{(R)}, w_{p, 0}^{(I)}, \cdots, w_{p, K-1}^{(I)}\right]^{T},
$$

where $w_{p, k}^{(R)}$ and $w_{p, k}^{(I)}$ are, respectively, the real and imaginary components of the complex scattering reflectivity $w_{p, k}$. A Gaussian distribution with mean zero and pixel-dependent variance $\alpha_{p}$, which is assumed to be independent of path $k$, is placed on $\check{\mathbf{w}}_{p}$, i.e.,

$$
\check{\mathbf{w}}_{p} \sim \mathcal{N}\left(\check{\mathbf{w}}_{p} \mid \mathbf{0}, \alpha_{p}^{-1} \mathbf{I}_{2 K}\right) .
$$

Note that this prior encourages group sparsity across $K$ paths through the shared parameters $\alpha_{p}$. Also, a Gaussian prior is placed on the additive measurement noise, i.e.,

$$
\check{\epsilon}_{m} \sim \mathcal{N}\left(\check{\epsilon}_{m} \mid \mathbf{0}, \beta_{0}^{-1} \mathbf{I}_{2}\right),
$$

where $\check{\epsilon}_{m}=\left[\tilde{\epsilon}_{m}^{(R)}, \tilde{\epsilon}_{m}^{(I)}\right]^{T}$. The posterior distribution of $\check{\mathbf{w}}$ is evaluated analytically based on Bayes' rule as,

$$
\operatorname{Pr}\left(\check{\mathbf{w}} \mid \mathbf{z}, \mathbf{A}\left(\mathbf{x}_{w}\right), \boldsymbol{\alpha}, \beta_{0}\right)=\mathcal{N}(\check{\mathbf{w}} \mid \boldsymbol{\mu}, \boldsymbol{\Sigma}),
$$

where

$$
\begin{aligned}
\boldsymbol{\mu} & =\beta_{0} \boldsymbol{\Sigma} \check{\mathbf{A}}\left(\mathbf{x}_{w}\right)^{T} \check{\mathbf{z}}, \\
\boldsymbol{\Sigma} & =\left(\beta_{0} \check{\mathbf{A}}\left(\mathbf{x}_{w}\right)^{T} \check{\mathbf{A}}\left(\mathbf{x}_{w}\right)+\left[\Gamma \otimes \mathbf{I}_{2 K}\right]^{-1}\right)^{-1}, \\
\boldsymbol{\Gamma} & =\operatorname{diag}\left(\alpha_{1}, \cdots, \alpha_{N_{x} N_{y}}\right), \\
\check{\mathbf{A}} & =\left[\begin{array}{cc}
\operatorname{Re}(\mathbf{A}) & -\operatorname{Im}(\mathbf{A}) \\
\operatorname{Im}(\mathbf{A}) & \operatorname{Re}(\mathbf{A})
\end{array}\right],
\end{aligned}
$$

with $\check{\mathbf{z}}=[\operatorname{Re}(\mathbf{z}), \operatorname{Im}(\mathbf{z})] \in \mathcal{R}^{2 J}$. Once the $\check{\mathbf{w}}$ are acquired, the complex scattering coefficient vector $\tilde{\mathbf{w}}$ is easily calculated.

By separately applying the CMT-BCS to the data with each set of hypothetic wall positions $\mathbf{x}_{w}^{(d)}=\left[x_{w_{1}}^{(d)}, \cdots, x_{w_{K-1}}^{(d)}\right], d=$ $1, \cdots, D$, we obtain respective complex scattering coefficient vectors as $\tilde{\mathbf{w}}^{(d)}, d=1, \cdots, D$. The common support of these $D$ estimated vectors is obtained as

$$
\mathbf{S}_{\tilde{\mathbf{w}}}=\operatorname{support}\left(\prod_{d=1}^{D} \tilde{\mathbf{w}}^{(d)}\right),
$$

where the support( $\cdot$ ) function returns a binary result for each pixel, i.e., it assumes the value 1 if the pixel takes a nonzero value, and 0 otherwise. The estimated true target position image is then obtained as the masked average of the $D$ individual images, expressed as,

$$
\hat{\mathbf{w}}=\left(\frac{1}{D} \sum_{d=1}^{D} \tilde{\mathbf{w}}^{(d)}\right) \circ \mathbf{s}_{\tilde{\mathbf{w}}} .
$$

Based on the estimated target reflection coefficient vector $\hat{\mathbf{w}}$, a maximum likelihood metric is introduced to estimate the wall position as,

$$
\hat{x}_{w_{l}}=\arg \min _{x_{w_{l}}}\left\|\mathbf{z}-\mathbf{A}\left(x_{w_{l}}\right) \hat{\mathbf{w}}\right\|_{2}^{2} .
$$

\section{B. Second Stage: High-Resolution Imaging}

Once the wall parameters $\hat{x}_{w_{l}}$ are obtained, we are able to construct the true sensing matrix. In the second stage, the CMT-BCS is reapplied to exploit multipath propagation, based on the multipath dictionaries constructed from the estimated wall locations, and acquire a high-resolution image. The flowchart of the proposed approach is shown in Fig. 2. 


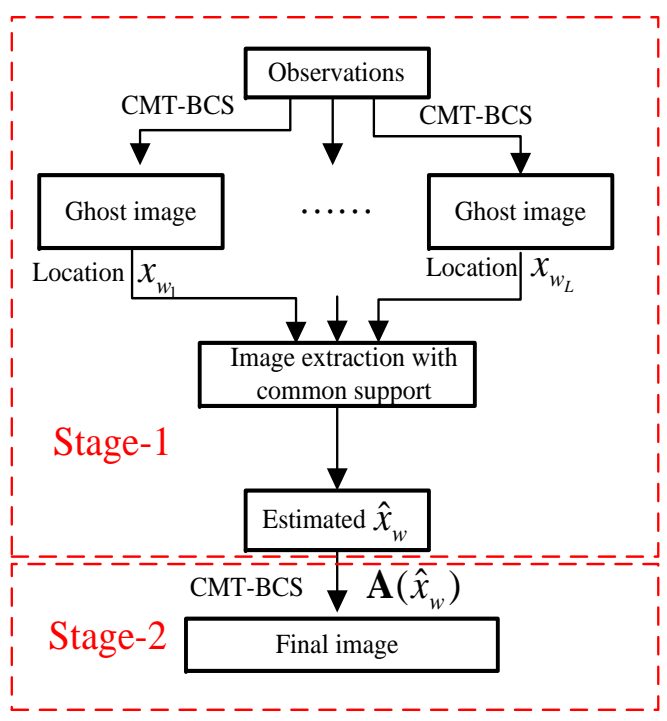

Fig. 2: Flowchart of the proposed algorithm.

\section{Simulation RESUlts}

The simulation parameters are selected as follows. A 40element monostatic ULA with an inter-element spacing of 2.5 $\mathrm{cm}$ and a stepped-frequency signal, consisting of 200 equally spaced frequency steps from $1 \mathrm{GHz}$ to $2 \mathrm{GHz}$, are used for imaging. The origin of the coordinate system is chosen to be at the center of the array. The front wall is located at $1 \mathrm{~m}$ downrange and its effects on signal propagation are assumed to have been perfectly compensated $[1,4]$. We consider two interior walls, i.e., the left and the right walls, which are respectively located at $x_{w_{1}}=-1.92 \mathrm{~m}$ and $x_{w_{2}}=1.93 \mathrm{~m}$ crossrange, respectively. The sparse scene is assumed to have 4 point targets, as shown in Fig. 3(a). The distance between two adjacent discretized image pixels along both crossrange and downrange is $0.075 \mathrm{~m}$. The received signal comprises the direct returns and the multipath returns via secondary reflections at the two interior walls, yielding a total of $K=3$ paths. The scattering coefficients for the direct paths are drawn from $\mathcal{C N}(0,1)$, and the signal amplitudes of the multipath returns are assumed to decay with the distance. Additive complex white Gaussian noise is added to the measurements with a signal-to-noise ratio (SNR) of $10 \mathrm{~dB}$. Only $25 \%$ frequencies are randomly selected at each antenna location for the CS-based imaging, whereas the full data set is used when applying the conventional back-projection algorithm.

Figs. 3(b)-3(d) show the reconstructed results of CMTBCS with $D=3$ sets of hypothetical wall locations. The true target positions are represented by green circles in each image. For the three cases, the hypothetical wall locations $\left\{x_{w_{1}}, x_{w_{2}}\right\}$ are chosen as $\{-2.10,1.90\} \mathrm{m},\{-2.08,1.95\} \mathrm{m}$, and $\{-1.95,2.05\} \mathrm{m}$, respectively. These values are chosen to span approximately half-wavelength at the lowest frequency for each wall position. Due to the inaccurate hypothetical wall locations, the assumed time delays do not match the true propagation delays for the paths involving secondary reflections and, as such, yield ghost targets that appear to be away from the true target positions. On the other hand, the true

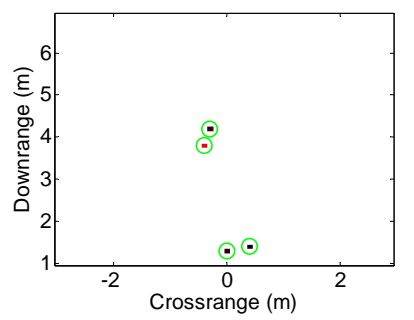

(a)

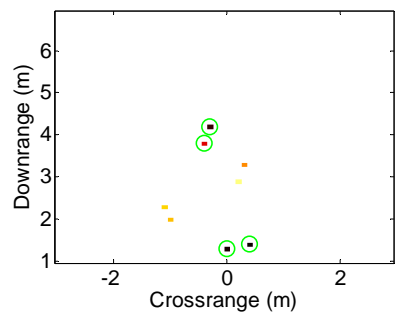

(c)

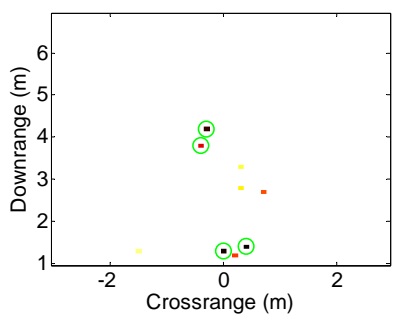

(b)

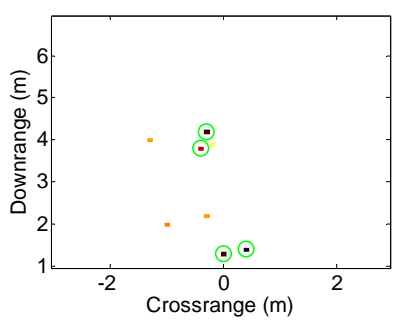

(d)
Fig. 3: Ground truth and three images obtained from CMT-BCS for three sets of hypothetical wall locations. (a) Original scene. (b) CMT-BCS result with $x_{w_{1}}=-2.10 \mathrm{~m}$ and $x_{w_{2}}=1.90 \mathrm{~m}$. (c) CMTBCS result with $x_{w_{1}}=-2.08 \mathrm{~m}$ and $x_{w_{2}}=1.95 \mathrm{~m}$. (d) CMT-BCS result with $x_{w_{1}}=-1.95 \mathrm{~m}$ and $x_{w_{2}}=2.05 \mathrm{~m}$.

targets are correctly estimated in these reconstructed images because the direct paths are not affected by the wall location errors.

Based on these reconstructed images, the true target positions are estimated as those that share a common support, as discussed earlier. The maximum likelihood metric in eq. (19) is then used to estimate the true wall locations, as shown in Fig. 4 , with a step size of $0.01 \mathrm{~m}$ for both side walls. We acquire the estimated left and right wall locations as $x_{w_{1}}=-1.94 \mathrm{~m}$ and $x_{w_{2}}=1.93 \mathrm{~m}$, respectively. Although the peaks in Fig. 4 appear to be periodic because of the wrapped phase, we are still able to acquire the wall location estimates with a high accuracy by using the maximum likelihood metric.

Subsequently, we construct the sensing matrix based on the estimated wall positions, and the CMT-BCS is reapplied to achieve the image shown in Fig. 5(a). Although the estimated wall locations are slightly biased, the time-delay deviations based on the estimated wall locations correspond to less than a pixel interval, thereby enabling acquisition of a ghostfree high-resolution image. To demonstrate the advantages of the proposed approach over the conventional through-the-wall imaging, we also show the results from the BP algorithm in Fig. 5(b). It is clear that, due to lack of proper multipath mitigation or exploitation, the resulting images are highly cluttered, even when the full data set is used for the BP-based imaging.

\section{CONClusion}

In this paper, a novel two-stage Bayesian compressive sensing approach was developed to perform through-the-wall radar imaging in the presence of interior wall multipath under inaccurate wall location information. In the first stage, we 


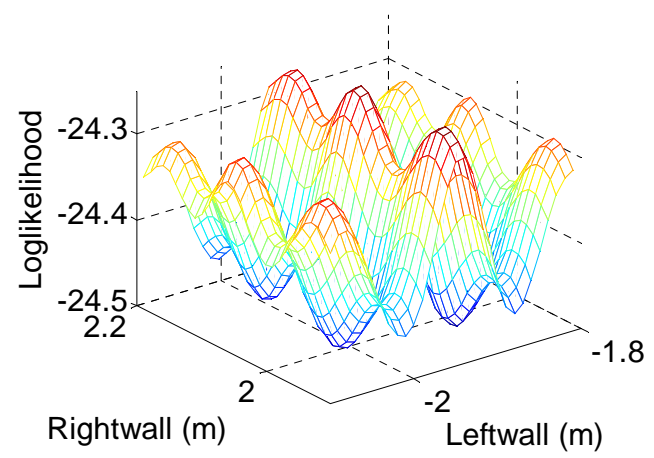

(a)

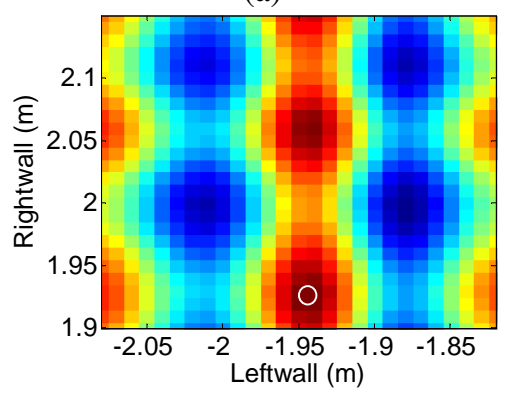

(b)

Fig. 4: Logarithm of likelihood in term of wall locations (a) 3D vision of logarithm of likelihood. (b) Logarithm of likelihood in term of left and right wall locations.

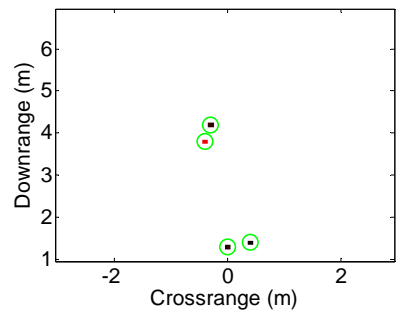

(a)

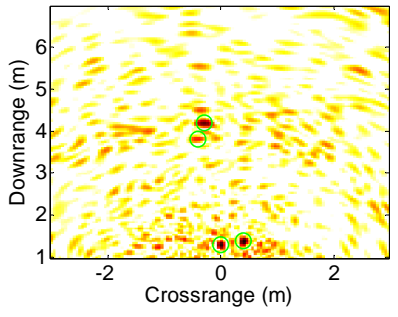

(b)

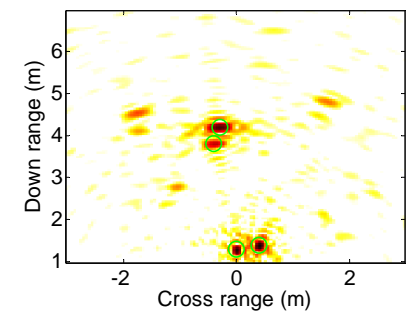

(c)

Fig. 5: Results in different approaches. (a) Proposed approach. (b) BP with partial data set. (c) BP with full data set.

generate several images with a small number of sets of hypothetical wall positions using the complex multi-task Bayesian compressive sensing approach, and the common support of the imaged scene is considered as the true positions of the targets. The wall positions are then estimated through maximum likelihood estimation. In the second stage, the CMT-BCS algorithm is used again to obtain the high-resolution image that exploits both direct and multipath propagation signals.
Simulation results are provided to validate the performance and superiority over conventional imaging techniques.

\section{REFERENCES}

[1] G. Wang, M. G. Amin, and Y. Zhang, "A new approach for target locations in the presence of wall ambiguity," IEEE Trans. Aerospace Electron. Syst., vol. 42, no. 1, pp. 301-315, 2006.

[2] Y. Zhang, M. G. Amin, and F. Ahmad, "Time-frequency analysis for the localization of multiple moving targets using dualfrequency radars," IEEE Signal Proc. Lett., vol. 15, pp. 777780, 2008.

[3] F. Ahmad and M. G. Amin, "Multi-location wideband synthetic aperture imaging for urban sensing applications," J. Franklin Inst., vol. 345, no. 6, pp. 618-639, 2008.

[4] M. G. Amin, Through-the-Wall Radar Imaging. CRC Press, 2011.

[5] M. G. Amin, Compressive Sensing for Urban Radars. CRC Press, 2014.

[6] P. Setlur, M. Amin, and F. Ahmad, "Multipath model and exploitation in through-the-wall and urban radar sensing," IEEE Trans. Geosci. Remote Sens., vol. 49, no. 10, pp. 4021-4034, 2011.

[7] S. Kidera, T. Sakamoto, and T. Sato, "Extended imaging algorithm based on aperture synthesis with double-scattered waves for UWB radars," IEEE Trans. Geosci. Remote Sens., vol. 49, no. 12, pp. 5128-5139, 2011.

[8] M. Leigsnering, F. Ahmad, M. Amin, and A. Zoubir, "Compressive sensing based specular multipath exploitation for throughthe-wall radar imaging," in Proc. IEEE ICASSP, pp. 6004-6008, Vancouver, Canada, May 2013.

[9] Q. Wu, Y. D. Zhang, M. G. Amin, and F. Ahmad, "Throughthe-wall radar imaging based on modified Bayesian compressive sensing," in Proc. IEEE China Summit and Int. Conf. Signal and Inform. Proc., Xi'an, China, pp. 232-236, July 2014.

[10] M. Leigsnering, F. Ahmad, M. G. Amin, and A. M. Zoubir, "Multipath exploitation in through-the-wall radar imaging using sparse reconstruction," IEEE Trans. Aerospace and Electronic Systems, vol. 50, no. 2, pp. 920-939, 2014.

[11] H. Mansour and D. Liu, "Blind multi-path elimination by sparse inversion in through-the-wall-imaging," in Proc. IEEE Int. Workshop on Comput. Adv. in Multi-Sensor Adaptive Process. (CAMSAP), Saint Martin, pp. 256-259, Dec. 2013.

[12] M. Leigsnering, F. Ahmad, M. G. Amin, and A. M. Zoubir, "CS based specular multipath exploitation in TWRI under wall position uncertainties," in IEEE Sensor Array and Multichannel Signal Proc. Workshop (SAM), pp. 481-484, A Coruna, Spain, June 2014.

[13] Q. Wu, Y. D. Zhang, M. G. Amin, and B. Himed, "Complex multitask Bayesian compressive sensing," in Proc. IEEE ICASSP, Florence, Italy, pp. 3375-3379, May 2014.

[14] Q. Wu, Y. Zhang, M. Amin, and B. Himed, "Multi-static passive radar SAR imaging based on Bayesian compressive sensing," in Proc. SPIE Compressive Sensing III Conf., vol. 9109, Baltimore, MD, May 2014.

[15] Z. Zhang and B. D. Rao, "Extension of SBL algorithm for the recovery of block sparse signals with inter-block correlation," IEEE Trans. Signal Proc., vol. 92, no. 7, pp. 1580-1590, 2012.

[16] Q. Wu, Y. D. Zhang, M. G. Amin, and B. Himed, "Mutlitask Bayesian compressive sensing exploiting intra-task dependency," IEEE Signal Proc. Lett., vol. 22, no. 4, pp. 430-434, 2015.

[17] S. D. Babacan, S. Nakajima, and M. N. Do, "Bayesian groupsparse modeling and variational inference," IEEE Trans. Signal Proc., vol. 62, no. 11, pp. 2906-2921, 2014.

[18] A. Gurbuz, J. McClellan, and W. Scott, "Compressive sensing for subsurface imaging using ground panetrating radar," Signal Process., vol. 89, no. 10, pp. 1959-1972, 2009. 